\title{
SUMMARY/PROGRESS REPORT
}

Mechanisms and Kinetics of Organic Aging and Characterization of Intermediates in High Level Waste

\section{Lead Principal Investigator:}

Donald M. Camaioni, Pacific Northwest National Laboratory, Environmental Molecular Sciences Laboratory, Richland, WA 99352, (509)375-2739, donald.camaioni@pnl.gov

\section{Co-Investigators:}

S. Tom Autrey, Pacific Northwest National Laboratory, Environmental Molecular Sciences Laboratory, Richland, WA 99352, (509)375-3792, tom.autrey@pnl.gov

Michel Dupuis, Pacific Northwest National Laboratory, Environmental Molecular Sciences Laboratory, Richland, WA 99352, (509)376-4921, michel.dupuis@ pnl.gov

Wendy Shaw, Pacific Northwest National Laboratory, Environmental Molecular Sciences Laboratory, Richland, WA 99352, (509)375-5922, wendy.shaw@pnl.gov

\section{Research Objective}

The objective of this project is to characterize significant chemical degradation pathways of organic chemicals in stored high-level waste and waste treatment streams. Recognizing that experiments cannot reproduce every conceivable scenario, the project works to develop predictive computational models of these chemistries. The effort is closely coordinated with a Notre Dame Radiation Laboratory project (EMSP No. 73832, “The $\mathrm{NO}_{\mathrm{x}}$ System in Nuclear Waste,” D. Meisel, Principal Investigator) that focuses on radiolytically-induced degradation of organic complexants in waste simulants. Investigators combine experimental observations, electronic structure computations, and theoretical methods development to achieve this goal. The resulting models facilitate accurate evaluation of hazardous conditions and support decision-making processes regarding safety, retrieval, treatment, and tank closure issues.

\section{Research Progress and Implication}

This report summarizes work after 6 years of the project, which started in 1998 and renewed in 2002. Progress in the following areas are described: 1) elucidating mechanisms of degradation of organic complexants and flammable gas generation, 2) measuring the thermochemistry and kinetics of reaction intermediates, 3) theoretically characterizing reaction intermediates, and 4) developing models for predicting rates of organic degradation and gas generation rates. In addition, interactions with Hanford site operators on site-specific issues concerning waste storage and treatment are highlighted.

Elucidation of Mechanisms of Degradation of Organic Complexants. At the start of the project, the investigators set out to model the thermal degradation of complexants in Hanford wastes. A mechanism had been advanced by Ashby et al. (ACS Sym Ser, 1994, 554, 247), in which aluminate ions catalyze the nitrosation of complexants. The resulting nitrite esters subsequently decompose to aldehydes that degrade to $\mathrm{H}_{2}$ and carboxylate ions in the strongly alkaline wastes. However, while attempting to elucidate this mechanism, the investigators obtained contradictory results (Camaioni and Autrey, ACS Sym Ser, 2000, 778,299 ; see also previous project reports). As depicted in Scheme 1, an alternative mechanism would first have $\mathrm{Al}(\mathrm{III})$ bind with glycolate anion and then have the complex react with nitrite ion. In this

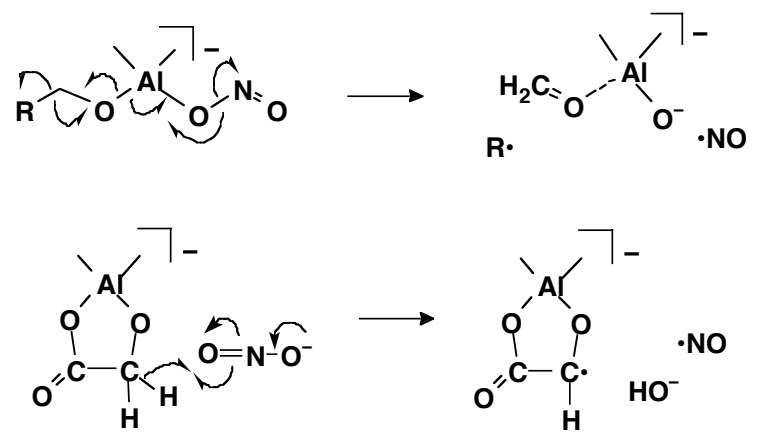
way, Al(III) would serve to lower the repulsive forces

Scheme 1 
between nitrite and anionic complexants, as well as be a bridge for electron transfer. Binding of $\mathrm{Al}(\mathrm{III})$ to the organic complexant may also activate adjacent $\mathrm{C}-\mathrm{H}$ and $\mathrm{C}-\mathrm{C}$ bonds.

The bottom reaction in Scheme 1 shows $\mathrm{H}$-atom transfer from an organoaluminate complex to nitrite ion. Analyses based on recent findings suggest that this path is preferred over the alternative of transferring the $\mathrm{H}$-atom to the nitrogen of nitrite ion. Using ethoxide ion as a model for glycolate ion, Scheme 2 shows the pathways for transfer of hydrogen to either the nitrogen or oxygen atoms of nitrite ion. Transfer to nitrogen generates the metastable ion, $\mathrm{HNO}_{2}{ }^{-}$, whereas transfer to oxygen is concerted with dissociation of the incipient $\mathrm{HONO}^{-}$to $\mathrm{NO}$ and $\mathrm{HO}^{-}$. $\mathrm{HNO}_{2}{ }^{-}(\mathrm{aq})$ is now known to form by addition of $\mathrm{H}$ atom to nitrite ion

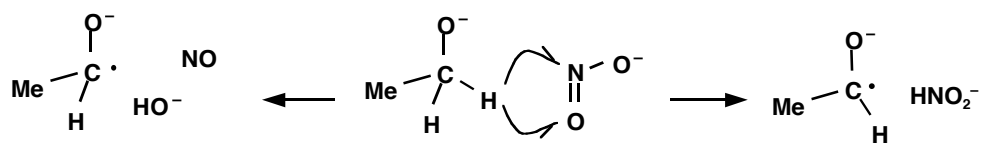
(Lymar et al. J. Phys. Chem. A, 2002, 106, 7245; Hug et al. J Phys

Scheme 2 Chem $A, \mathbf{2 0 0 4}$, in press). $\mathrm{HNO}_{2}^{-}(\mathrm{aq})$ deprotonates above $\mathrm{pH} \sim 6$ to give another metastable species, $\mathrm{NO}_{2}{ }^{2-}$ (aq), which undergoes general acid-catalyzed decomposition to $\mathrm{NO}$ and water (and $\mathrm{B}^{-}$). Protonation of $\mathrm{NO}_{2}{ }^{2-}$, presumably at oxygen, induces decomposition to $\mathrm{NO}$ and $\mathrm{OH}^{-}$, as the isomer $\mathrm{HONO}^{-}$is not detected in solution. The related metastable ion, $\mathrm{NO}_{3}{ }^{2-}$, behaves similarly, decomposing to $\mathrm{NO}_{2}$ and $\mathrm{OH}^{-}$ on protonation by general acids (Cook et al. J Phys Chem A, 2001, 105, 3658). Consistent with these observations, computations show that dissociation of $\mathrm{HONO}^{-}$to $\mathrm{OH}^{-}$and $\mathrm{NO}$ is a barrierless process in water. Addition of $\mathrm{H}$ atom to nitrite ion in water is computed to be exergonic by $28 \mathrm{kcal} / \mathrm{mol}$. From this value and the $D G^{\circ}=82 \mathrm{kcal} / \mathrm{mol}$ for the $\alpha-\mathrm{C}-\mathrm{H}$ in ethoxide ion, ${ }^{1}$ hydrogen atom transfer to the nitrogen of $\mathrm{NO}_{2}^{-}$(right hand reaction in Scheme 2) is endergonic by $44 \mathrm{kcal} / \mathrm{mol}$. In contrast, the reaction on left of Scheme 2 is exergonic by $\geq 4 \mathrm{kcal} / \mathrm{mol}$. It remains to compute the transition structure and energetics for this reaction and to determine whether complexation with $\mathrm{Al}(\mathrm{III})$ may lower the activation barrier and thereby demonstrate the catalytic role of aluminum ion.

Thermochemistry and Kinetics of Intermediates. The project is using photoacoustic calorimetry (PAC) to gain fundamental knowledge as how $\mathrm{Al}(\mathrm{III})$ may activate organic complexants to undergo redox reactions. The PAC technique is based on the phenomenon that chemical reactions induced by absorption of a light pulse produce a measurable sound pulse that is relatable to the enthalpy changes, volume changes, and rates of the induced reactions. A power of the technique is that such information can be used to determine bond dissociation energies (BDEs) in solution. The mechanisms discussed above imply that the $\mathrm{C}-\mathrm{H}$ bond of an $\mathrm{Al}(\mathrm{III})$ glycolate complex may be activated relative to the bond in uncomplexed glycolate ion. If the $\mathrm{C}-\mathrm{H}$ BDE of the $\mathrm{Al}(\mathrm{III})$ glycolate complex is lower than the C-H BDE of free glycolate ions then the activation barrier for hydrogen atom transfer to nitrite may be lowered by $\mathrm{Al}(\mathrm{III})$ complexation.

As a first step toward obtaining aqueous thermochemistry of organic radicals in water, the researchers adapted the PAC technique to use hydrogen peroxide as a photo-precursor and measured the thermochemistry and volume changes for the key reactions:

$$
\begin{aligned}
\mathrm{HOOH}(\mathrm{aq}) & \rightarrow 2 \mathrm{OH}(\mathrm{aq}) \\
\mathrm{OH}(\mathrm{aq})+\mathrm{HOOH}(\mathrm{aq}) & \rightarrow \mathrm{OOH}(\mathrm{aq})+\mathrm{H}_{2} \mathrm{O}(\mathrm{l})
\end{aligned}
$$

From these measurements they determined the enthalpies of formation, the enthalpies of hydration, and partial molar volumes of $\mathrm{OH}(\mathrm{aq})$ and $\mathrm{OOH}(\mathrm{aq})$. These results ${ }^{2}$ constitute the first measurements of their

\footnotetext{
${ }^{1}$ from $D G^{\circ}=88 \mathrm{kcal} / \mathrm{mol}$ and $\mathrm{p} K_{\mathrm{a}}=15.7$ for aqueous ethanol and $\mathrm{p} K_{\mathrm{a}}=11.5$ for hydroxyethyl radical (Schwarz and Dodson, J Phys Chem 1989, 93, 409) .

${ }^{2}$ Results for reaction (1) are published: Autrey et al. J Am Chem Soc 2004, 126, 3680. A manuscript reporting the results for reaction (2) is in preparation.
} 
partial molal volumes. Such measurements are useful for predicting the pressure dependence of reactions and provide insight to the interactions of solute with water. Overall, the results provide confidence in the pulsed PAC technique for measuring aqueous thermochemistry and open the way to obtaining thermochemistry for radicals that can be formed by reaction of $\mathrm{OH}$ with aqueous substrates.

Experiments to obtain the thermochemistry of glycolate ion are in progress. Figure 1 shows that, with glycolate added to solutions of $\mathrm{H}_{2} \mathrm{O}_{2}$, a PAC response is observed for the reaction:

$$
\mathrm{OH}(\mathrm{aq})+\mathrm{HOCH}_{2} \mathrm{CO}_{2}^{-}(\mathrm{aq}) \rightarrow \mathrm{H}_{2} \mathrm{O}(\mathrm{l})+\mathrm{HOCHCO}_{2}^{-}(\mathrm{aq})
$$

The amplitude of the signal increases with increasing glycolate, which suggests that more heat is being released and/or the reaction volume is more positive for reaction (3) compared to reaction (2). It remains for the researchers to measure the temperature dependence of the PAC signal to determine the reaction volume, as this will provide the volume contribution to the signal and allow the BDE of glycolate ion in water to be determined. Subsequent work will then focus on determining the BDE of the Al(III)glycolate complex. Comparison of results will then show how $\mathrm{Al}$ complexation affects the stability of the glycolate radical.

Figure 1. Photoacoustic signals from $0.2-\mathrm{M} \mathrm{H}_{2} \mathrm{O}_{2}$ solutions containing $0(--), 5(--)$, and $10(--) \mathrm{mM}$ sodium glycolate. Deconvolution of the time dependence of the signal gives the psuedo-first order rate constant for reactions of $\mathrm{HO}$ radical. The inset shows a plot and least squares fit of the observed first-order rate constant vs. glycolate concentration. The slope equals the secondorder rate constant for reaction with glycolate and the intercept equals the reaction with $\mathrm{H}_{2} \mathrm{O}_{2}$ plus other background reactions. Rate constants are in agreement with literature values.

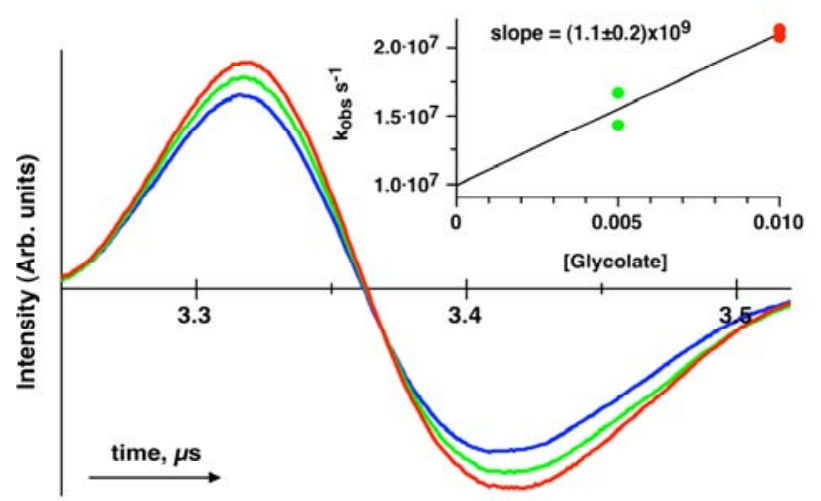

Theoretical Characterization of Intermediates in Aqueous Solution. In support of the experimental objectives and model development work, the investigators developed a new cavity definition protocol that yields accurate solvation energies and electrode potentials for selected oxoanions, $\mathrm{XO}_{\mathrm{m}}{ }^{\mathrm{n}-}$, including some for which other cavity protocols do not perform well enough (Camaioni, et al. J Phys Chem A, 2003, 107, 5778). In this new definition scheme with cavities made up of interlocked atomic spheres, the radii are given by simple empirically-based expressions involving effective atomic charges of the solute atoms that fit the solute molecular electrostatic potential and a bond length-dependent factor to account for atomic size and hybridization. The scheme shows substantial qualitative differences with other previously proposed schemes, for example by assigning a large radius to the central atom of the oxoanions. This difference is put on a firm theoretical basis in the case of $\mathrm{NO}_{3}{ }^{-}$through an analysis of the molecular electrostatic potential of the $\mathrm{NO}_{3}{ }^{-}$and an analysis of its interaction with a 'solvent' water molecule (see Figure 2). From these results the investigators surmise that the solvent molecules remain further away from the nitrogen atom, a finding consistent with the large radius assigned by the new scheme for nitrogen. The same qualitative feature holds true for the ten oxoanions and six neutral oxides they considered.

Work is now directed toward extending the protocol to oxohydroxoanions, $\mathrm{HOXO}_{\mathrm{m}}{ }^{\mathrm{n}-}$, and corresponding neutral species which are also relevant to tank waste chemistry. Supported by analyses such as in Figure 2, the researchers assert that solute cavities used in continuum solvation theories should reflect specific (hydrogen bond) solute-water interactions. To gain insight to the shape of cavities for solutes with $\mathrm{OH}$ functionality, the researchers have defined cavities for some representative solutes from ab initio calculations of some solute interacting with a water molecule. The approach "rolls" a water 


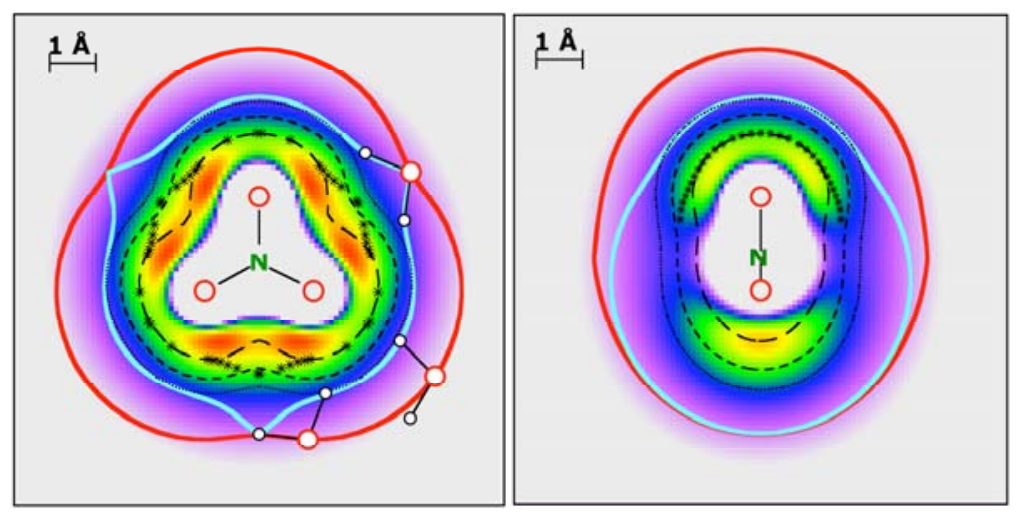

Figure 2. Colored-coded maps show electrostatic potential and electron density information for $\mathrm{NO}_{3}{ }^{-}$superimposed on $\mathrm{H}_{2} \mathrm{O}-\mathrm{NO}_{3}{ }^{-}$minimum interaction energy contours: (left) view of the molecular plane and (right) view of the plane perpendicular to the molecular plane along one $\mathrm{N}-\mathrm{O}$ bond. Electron isodensity contours values: $\cdots 5 \times 10^{-4},---2 \times 10^{-3}$, $---1 \times 10^{-2} \mathrm{e}^{-} / \mathrm{au}^{3}$; Electrostatic isopotential contour values $(\mathrm{kcal} / \mathrm{mol})$;

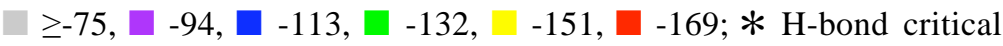
points; (red line) water-oxygen minimum interaction energy surface; (blue line) water-hydrogen minimum interaction energy surface. Reproduced from Camaioni et al. J Phys Chem A, 2003, 107, 5778. Copyright () 2003 American Chemical Society.

molecule over the solute molecule to define the trough of minimum energy in the solute-water interaction. A 3-dimensional electron density corresponding to a selected isodensity contour of an isolated water molecule is further attached to the rolling water solvent molecule in all of its positions in the trough. The cavity is then defined as the inside envelop of the 'rolling density'. Figure 3 shows the procedure as it is applied to define the cavity for $\mathrm{OH}$ in water (Autrey et al. ibid). Other solutes to which the technique has been applied include $\mathrm{H}_{2} \mathrm{O}, \mathrm{H}_{2} \mathrm{O}_{2}, \mathrm{HO}_{2}$, and $\mathrm{NO}_{3}^{-}$. The cavities generated in this way have in common that the continuum approaches more closely to regions where hydrogen bonding with water is more favored. Interestingly, the same isocontour value $\left(0.073 \AA^{3} / a\right.$.u. $)$ for the "rolling" water density exactly reproduces the experimental hydration free energies of all the solutes (within experimental uncertainties). Thus, though this technique is tedious and time consuming, it shows promise for accurately predicting hydration energies of solutes. The technique warrants further work to apply it to a variety of solutes to gain deeper understanding of how to improve the accuracy of continuum solvation models.

The results from defining cavities by this technique (Figure 3) suggest that in defining cavities of interlocking spheres for solutes with oxo and hydroxo groups, both the oxygen and hydrogen radii may be correlated with the effective charge on the oxygen of the $\mathrm{OH}$ group. Preliminary work to define the optimum cavity radii for $\mathrm{OH}$ and $\mathrm{OH}^{-}$shows that when the radius of $\mathrm{O}$ is set proportional to the effective charge on $\mathrm{O}$, the radius of $\mathrm{H}$ then varies inversely with the charge. Having established this relationship, work to define the cavity radii for $\mathrm{OH}$ groups in oxohydroxo systems in is progress.

Modeling $\mathrm{H}_{2}$ Generation Rates in the Hanford Waste Treatment and Immobilization Plant. The PI worked with Bechtel National, Inc., Research and Technology Department to provide valuable insight and design tools for reliably bounding $\mathrm{H}_{2}$ generation rates in the waste treatment and immobilization plant (WTP). The work assessed the processing steps which would most affect $\mathrm{H}_{2}$ generation by the waste and recommended which of the effects could be modeled with existing information and which would require laboratory testing. Important issues that were identified included the effects of beta/gamma and alpha radiolysis and of adding hydroxide, permanganate, air, sugar and glass-forming materials at various stages 
in the processing prior to the vitrification step. Water radiolysis was determined to be a significant source of $\mathrm{H}_{2}$ following pretreatment of high level wastes. Fundamental information obtained from recent studies of the mechanism of water radiolysis by LaVerne and Pimblott (see: J Phys Chem A, 2000, 104, 9820), which was funded by programs in the DOE's Office of Science, enabled the development of accurate, yet simple equations for predicting rates of $\mathrm{H}_{2}$ generation from water radiolysis in the WTP process streams. Bechtel National, Inc. was guided by the information in the reports and used the equations in calculations to evaluate air purging and mixing rate design requirements for vessels that will be installed in the plant.

As a result of this work, the DOE Office of River Protection, who oversees the storage and retrieval of Hanford wastes, requested that this new information be incorporated in models of $\mathrm{H}_{2}$ generation in the stored (tank) wastes. Camaioni is collaborating with Albert $\mathrm{Hu}(\mathrm{CH} 2 \mathrm{M}$ Hill Group, Hanford) to revise the Hanford site's model for predicting $\mathrm{H}_{2}$ generation in the waste storage tanks.

\section{Planned Activities}

In the remaining months of the current project, the investigators will work to finish measurements of the thermochemistry and volumetric properties of aqueous reactions of $\mathrm{OH}$ radical with glycolate ion and start work on aluminum(III)glycolate complexes. The computational effort will work to extend the new cavity definition protocol to include solutes with hydroxyl and alkyl groups. Extensions to multiplycharged anions may also be investigated. Two Summer University Laboratory Intern undergraduate students (Mr. Aaron K. Brown, Univ. of Washington, and Mr. Quinn Gilcrease, Western Washington University) have been hired to help with this work. Computations efforts will start on determining the activation barriers for reactions in Scheme 1 with and without $\mathrm{Al}(\mathrm{III})$. Manuscripts on the thermochemisty and volumetric properties of $\mathrm{HO}_{2}$ and glycolate radicals in water will be prepared.

A renewal proposal has been submitted. If renewed, the project will continue from where the current project leaves off, working to further advance understanding of significant chemical reactions that occur in high-level wastes, and computational capabilities to accurately model that chemistry.

\section{Information Access (since 2001)}

\section{Publications:}

1. "EPR Detection of $\mathrm{HNO}_{2}$ ' in the Radiolysis of Aqueous Nitrite and Quantum Chemical Calculation of its Stability and Hyperfine Parameters," G. L. Hug, D. M. Camaioni, I. Carmichael, J. Phys. Chem. A, 2004, accepted for publication. (with EMSP project no. 73832)

2. "Thermochemistry of Aqueous Hydroxyl Radical from Advances in Photoacoustic Calorimetry and ab Initio Continuum Solvation Theory," T. Autrey, A. K. Brown, D. M. Camaioni, M. Dupuis, N. S. Foster, A. Getty, J. Am. Chem. Soc. 2004, 126, 3680-3681.

3. "Theoretical Characterization of Oxoanion, $\mathrm{XO}_{\mathrm{m}}{ }^{\mathrm{n}-}$, Solvation," D. M. Camaioni, M. Dupuis, and J. Bentley, J. Phys. Chem. A 2003, 107, 5778-5788. (with EMSP project no. 73832).

4. "Reducing Radicals in Nitrate Solutions. The $\mathrm{NO}_{3}{ }^{-}$System Revisited," A. R Cook, N. Dimitrijevic, B. W. Dreyfus, D. Meisel, L. A. Curtiss, and D. M. Camaioni. J. Phys. Chem. A, 2001, 105, 3658-3666. (with EMSP project no. 73832).

\section{Reports in Collaboration with DOE Site Contractors:}

1. Gas Generation Testing and Support for the Hanford Waste Treatment and Immobilization Plant, Bryan, S. A.; D. M. Camaioni, T. G. Levitskaia, B. K. McNamara, R. L. Sell, L. M. Stock. 2004. WTP-RPT-115 Rev. 0, Battelle, Pacific Northwest Laboratories, Richland, Washington.

2. Decomposition Pathways For The Savannah River Site Caustic-Side Solvent Extraction System, Stock, L. M.; D. M. Camaioni, D. Wester, and D. Gutsche. 2002. PNNL-13935, Pacific Northwest National Laboratory, Richland Washington. 